\title{
'Step by step we build up a rapport'
}

\author{
By Kate Quinlan
}

Is it realistic to see all patients by the age of one, as

advocated by the British Society of Paediatric Dentistry?

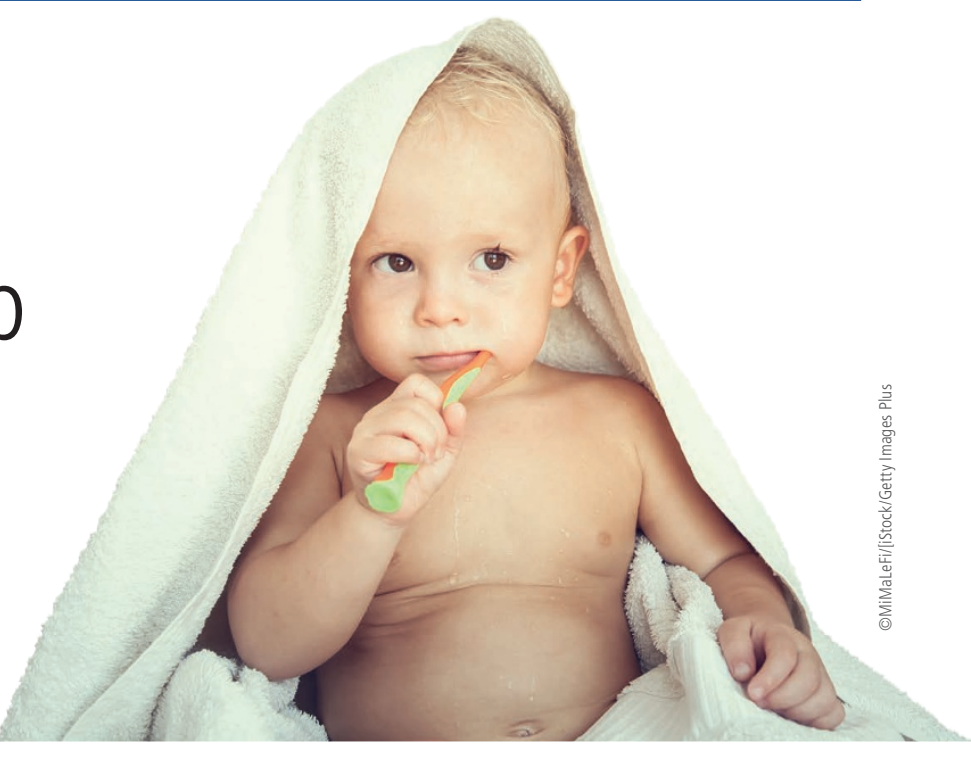

$\mathrm{I}$ $\mathrm{n}$ the previous issue of the $B D J$ it was reported that the British Society of Paediatric Dentistry (BSPD) is campaigning to make 'Dental Check by One' the norm for all children in the UK, to reduce dental caries and the number one cause of children being admitted to hospital. ${ }^{1}$

Dr Claire Stevens, Consultant in Paediatric Dentistry and Vice-President of BSPD, has spoken to delegates at both the British Dental Conference and Exhibition in Manchester and the Local Dental Committees Annual Conference in Birmingham in recent months, urging dentists to try and see four additional children under age two in the coming year.

Data from the Faculty of Dental Surgery (FDS) at the Royal College of Surgeons released last month showed that approximately $80 \%$ of children between the age of one and two in the 12 months leading up to 31 March 2017 did not visit an NHS dentist, ${ }^{2}$ despite guidance saying children should start dental check-ups when they develop their first tooth. The Faculty's analysis also found that almost $60 \%$ of children aged one to four did not have a dental check-up in the same period.

During 2015/16, there were 9,220 cases of tooth extractions performed in hospitals on children aged one to four, many of which can be attributed to tooth decay, which is $90 \%$ preventable. $^{2}$

In a statement, the British Dental Association (BDA) said that it had conducted polling indicating that millions of UK parents are unaware that NHS dentistry is free for children under 18 , and has called on all parties to deliver an oral health strategy based on prevention.
I spoke to a number of general dental practitioners to elicit their views on treating child patients in their practices, and their response to the BSPD's call to see all children by age one.

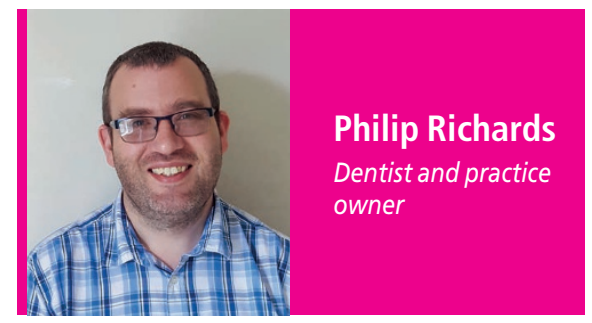

Type of practice: NHS/private: private adults, NHS children's list.

Do you accept new child patients at your practice? Yes.

At what age do you accept new child patients? From birth if the parents wish. The earlier I can get the opportunity to get a child comfortable in the dental environment the better.

How do you approach a first appointment with a young child? It is very much led by the child's personality. My goal is largely to get them used to the environment: the sights, sounds and smells of a dental practice can be very strange. Getting a full examination done is secondary as this first appointment will lay the groundwork for all future contacts.

I take time to talk to the child as much as possible, depending on their age and level of understanding. I show them how the chair works etc.

What do you discuss with a child's parent/guardian at that first appointment? Basic oral hygiene, diet advice etc, depending on the patient's needs.
What do you do if the young child refuses to cooperate/open their mouth? I obviously try to persuade them to open and give lots of encouragement and praise for whatever is achieved. If we are getting nowhere I don't persist for long as upsetting the child never works and will just make the next appointment harder. In that situation I will make another appointment for a second attempt a few months later, which will allow the parents time to talk to their child and prepare them. Stickers and other motivational aids can be useful.

Recall time for young child patient check-ups at your practice: Between three and six months depending on the child's needs.

Do you agree with the BSPD campaign to make 'Dental Check by age One' the norm for all children in the UK? Do you think it is realistic to see all patients by age one? I agree completely with the campaign. I actually find treating children very challenging, and it is not at all my favourite aspect of dentistry. Any method of preventing dental problems in this group is really worth pursuing, and in the cases where treatment becomes necessary it is far better that the child is being looked after in an environment that they know and are comfortable in rather than it being their first visit to a strange place, when they are already distressed from toothache.

Any other comments: In the past I have also worked as a dentist on hospital GA lists. If anyone has any doubt about the need for getting to youngsters and their parents early to provide suitable preventative advice and the correct interventions then they need to go and observe one of those lists. 


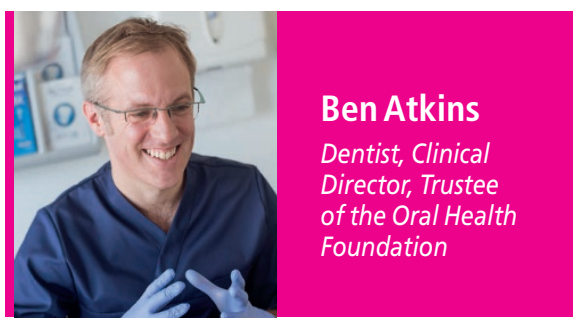

Type of practice: (multiple in our case): NHS/private (based on NHS practice with 95\% NHS).

Do you accept new child patients at your practice? Our practices fully accept new NHS patients (have done for the past 18 years), approximately 400 per month over three sites.

How do you approach child patients? I think treating adults like kids and kids like adults can't go far wrong.

I have developed my own 'patient journey' for an examination which I would be happy to share with $B D J$ readers.

What do you discuss with a child's parent/guardian at their first appointment? To be fair usually I explain to the parent that I won't be talking to them, I will be explaining treatment/prevention/what I am going to do directly to the child - as the more control I can give to the child the better.

Depending on the time of the patient journey this really is important. If the child is months old, I discuss with the parent the aim of this initial journey is to get the patient eventually to jump onto the chair of their own accord and not need any treatment.

I will outline behaviour setting patterns, like when to introduce a toothbrush, diet patterns (fruit not sweets, water not juice, milk not juice, sweet days etc).

I relate stories to my experience as a parent.

Generally, I provide information, information, information.

If the patient is in pain, ie too late for a really gentle approach, pain removal is key then slowly building up their confidence.

What do you do if the young child refuses to cooperate/open their mouth?

I consider: 'Does it matter?' 'What are the siblings like?' 'Are they in pain?'

If low risk then we will leave it for next time, or simply book another appointment; after a few appointments the patient will generally trust you.

If the patient is in pain, one of the best tricks is to simply explain to the parents we need to look into their mouth and ask them to sit outside whilst you see the next patient (by this time the patient generally has had a discussion with the parent and is a lot more cooperative - you are pushing the responsibility onto the parent).

Do you agree with the BSPD campaign to make 'Dental Check by age One' the norm for all children in the UK? Yes yes yes. Really, I never understand why some patients are not seen before this age.

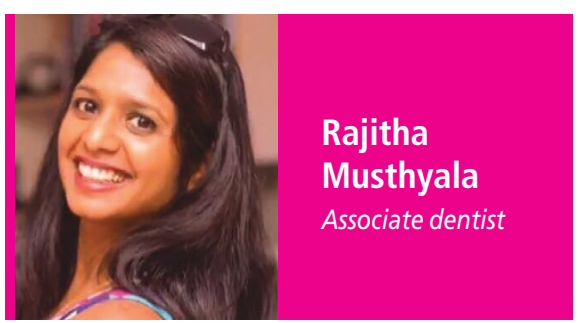

Type of practice: Mixed.

At what age do you accept new child patients? I accept children from age six weeks onwards.

What do you discuss with a child's parent/guardian at their first appointment? I talk to the child's mother about breastfeeding/bottle feeding, brushing as soon as a tooth erupts and provide fluoride advice. I establish sibling history and the patient's social economic status and their motivation towards prevention of decay. I encourage parents to give their children clear water for drinks rather than diluting juices, and to give them fresh fruit and vegetable sticks followed by cheese as snacks. I point out the hidden sugars in ready-made snacks.

What do you do if the young child refuses to cooperate/open their mouth? I don't force them if they are not comfortable, but I blow up a glove balloon (Fig. 1) and draw teeth and a face on them. I ask the child how the lion roars and have a quick glance at their teeth. I will tell them the chair is a like a seesaw in the park and I will give them a ride. I offer stickers and sippy cups and brushes for the child to choose.

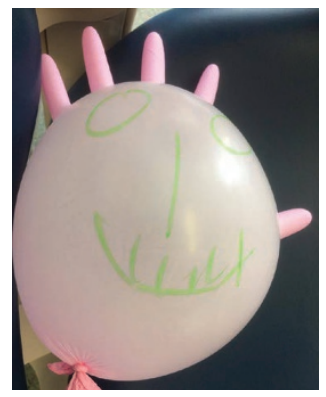

Fig. 1 'Glove balloon' used with child patient
Even if a child is scared at the first appointment, they often respond at the second or third.

Any other comments: I encourage all my pregnant patients to register their children as soon as they are born. We also encourage parents to download the Be Food Smart $\mathrm{app}^{3}$ which works with the traffic light food labelling system.

Here in Scotland the Childsmile scheme helps and we have Childsmile nurses for children who need more time or help with their oral health.

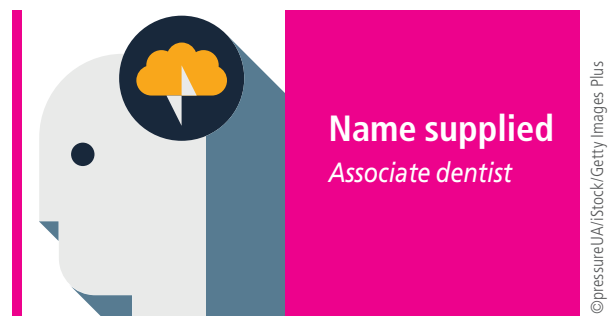

Type of practice: Mixed.

Do you accept new child patients at your practice? No - full list at present, although if an existing patient has a baby then we agree to take them on.

At what age do you accept new child patients? As soon as patients want to bring them - we always give mum advice for the child and suggest she brings baby to each of her appointments to get the child used to being in the surgery.

How do you approach a first appointment with a young child? Light touch. Sit child on parent's knee on the dental chair (or other surgery chair if the child is apprehensive about the main chair). Talk to parents (and if child is old enough to understand then with the child also). Ask child to say 'ah' and use pen torch to look in the mouth. This appointment is about building confidence and delivering education measures.

What do you discuss with a child's parent/guardian at that first appointment? Diet mainly and oral health.

What do you do if the young child refuses to cooperate/open their mouth? No problems - talk to them and ask them to check out Peppa Pig on YouTube ahead of the next appointment to learn about what you normally do at a dental appointment. There's no point putting the child off before you've even started. Normally though, I can get at least a small look in the mouth after having had a long chat. I also use mirroring by opening my own mouth and getting parents to do likewise and allowing me to look in their mouth with the pen 
1 torch - kids usually copy even if only for a very small look. At this point lots of praise and then reinforce educational messages.

Recall time for young child patient check-ups at your practice: Six months unless high risk - eg sibling high caries rate or blackcurrant juice in a bottle when they enter the surgery - in that case three month checks.

Do you agree with the BSPD campaign to make 'Dental Check by age One' the norm for all children in the UK? If there was a surplus of supply of NHS dental care, meaning it's available (and maybe even a choice!) for anyone who wants it, then it would be a great idea. But only providing sufficient funding to see just over half of the population tells you that this isn't the case. There is a severe shortfall in supply in this area. So no, it is a bad idea and risks developing resentment from adult patients who have great need but can't access care.

Do you think it is realistic to see all patients by age one? As a marketing plan for getting families through the door it is a great idea. But if we are asking people to bring their under ones whilst telling them that we can't see anyone else in the family unless they pay privately then this will not go down well. Look at it from a busy mother's perspective. She has a six-month-old baby and we say of course we'll see your child who is only just getting their first tooth, but awfully sorry your toothache can only be seen at the local urgent dental care clinic as we don't have any space here for new adult patients.

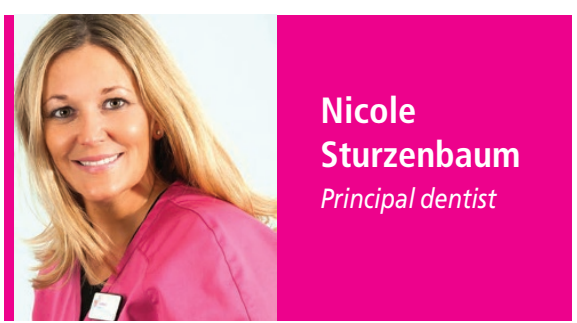

Type of practice: Children's private practice.

Do you accept new child patients at your practice? Yes.

At what age do you accept new child patients? From six months onwards, or earlier if needed.

How do you approach a first appointment with a young child? Very young children (under 18 months) are often quite easy to deal with, especially with our TVs on the ceiling (showing child friendly cartoons). If the children are already at an age where they can communicate (2+), then we use our puppet, with the tell, show and do approach.

What do you discuss with a child's parent/ guardian at that first appointment? Medical history (risk factors), brushing habits, diet (bottle feeding, breastfeeding) and we do a tooth brushing school if the children are cooperative enough/showing the parents how to brush. It's all about educating the parents and the child to prevent decay and giving the children a positive attitude towards their teeth and the dentist.

What do you do if the young child refuses to cooperate/open their mouth? If the children are very young we do the exam on the

knee of the parents, which usually works well. If the children are older we give them choices of where to have the exam (maybe on a normal chair instead of the dental chair) or we let them do the exam on mum or a doll first. Step by step we built up a rapport. Behaviour management techniques in different ways should be in constant use when approaching a child in the dental chair and all dentists should have basic training in it.

Recall time for young child patient check-ups at your practice: $3-6$ months depending on the caries risk.

Do you agree with the BSPD campaign to make 'Dental Check by age One' the norm? Do you think it is realistic to see all patients by age one? Yes, I agree with the campaign and I think it is realistic.

Any other comments: I support the campaign and think with campaigns like this it's all about changing the attitude of parents towards the dental need of their children. At the moment it's all about treating teeth if pain or damage already has happened, but what we need is prophylaxis, so all children see their dentist on a regular basis to prevent decay/ damage and anxiety in the first place.

1. BDJ Upfront. UK dentists urged to see children by age one. Br Dent J 2017; 222: 916.

2. Royal College of Surgeons. $80 \%$ of 1 to 2 -year-olds didn't visit an NHS dentist last year. 8 June 2017. Available at: https://www.rcseng.ac.uk/news-and-events/media-centre/ press-releases/toddler-dental-vist-story/ (accessed June 2017)

3. change4life. Be Food Smart app. Available at: https://www nhs.uk/change4life-beta/be-food-smart\#zylivYehMhd8goA4.97 (accessed June 2017).

\section{Honours, awards, appointments}

\section{Queen's Birthday Honours}

Five dentists have received awards in the Queen's Birthday Honours list:

\section{$O B E$}

Ms Dawn Ailsa Adams, Clinical Director, Public Dental Service, NHS Fife. For services to dentistry.

Professor Nigel Peter Hunt, Head of Orthodontic Department, Eastman Dental Institute, University College London. For services to dentistry and orthodontics.

Mrs Margaret Katherine Ross, lately Senior Lecturer for Dental Care Professionals, University of Edinburgh. For services to dentistry.

$M B E$

Dr Serbjit Kaur, dentist, Leicestershire. For services to dentistry.

\section{BEM}

Dr Linda Helene Greenwall, dentist, Hampstead Healthcare Ltd. For services to the dental profession in the UK and abroad.

Workshop for new writers and peer reviewers

The $B D J$ will once again be supporting an all day workshop of the British Dental Editors Forum for new writers and peer reviewers. New this year will be a separate afternoon session for those specifically interested in peer review. The workshop will be held on Wednesday 25 October 2017 at the BDA, 64 Wimpole

Street, London, W1G 8YS. The cost is $£ 40$ ( $£ 20$ for full-time students) to include lunch and refreshments and will attract five hours of CPD. Further details are available from Stephen Hancocks (stephen.hancocks@bda.org). 